\title{
A COMPARATIVE STUDY OF PRIMARY REPAIR VERSUS RESECTION AND ANASTOMOSIS IN JEJUNOILEAL PERFORATIONS
}

\section{General Surgery}

Dr. Shivaprasad D. MBBS, MS,DNB, General Surgery, Assistant Professor, Department Of General Surgery S

\author{
, JSS Hospital , Mysuru.
}

\section{Dr. Thrishuli P.B*}

MBBS MS General Surgery, Professor , Department Of General Surgery, JSS Hospital, Mysuru. *Corresponding Author

\begin{tabular}{ll}
\hline Dr. Deva & MBBS, Junior Resident General Surgery, Department Of General Surgery, JSS Hospital, \\
Keerthana D. Y & Mysuru.
\end{tabular}

\section{ABSTRACT}

INTRODUCTION: Ileo-jejunal perforation is the fifth common cause of acute abdomen presenting as emergency(1). Despite availability of modern diagnostic facilities and advances in treatment regimes, the abrupt onset and rapid downhill course of disease has a high mortality rate. $(1,2)$ AIMS AND OBJECTIVES: To contribute in the betterment of knowledge in the management of ileo-jejunal perforations and to compare and analyse the primary repair (vs) resection and anastomosis in ileojejunal perforation in terms of post -operative morbidity and mortality. METHOD: This was a prospective observational study conducted in JSS HOSPITAL,Mysuru .A total of thirty patients admitted with ileojejunal perforations during a period of one and half years undergoing either primary closure or resection and anastomosis were taken for the study RESULTS: Trauma ( Blunt trauma ) was the most common cause of ileo jejunal perforation in this observational study followed by enteric perforations. Most common complication following surgery was identified to be wound infection/ dehiscence(27.6\%) followed by respiratory complications(13.3\%). CONCLUSION: Post operative complications and duration of hospital stay were less with primary closure group and hence primary closure should always be considered as first option whenever conditions are apt

\section{KEYWORDS}

Perforation, Primary Closure, Resection And Anastamosis.

\section{INTRODUCTION:}

Peritonitis caused secondary to perforation of small intestine is one of the most common abdominal emergency ${ }^{(3)}$. Small bowel perforations are broadly classified into traumatic and spontaneous. Spontaneous perforation in the ileum can be due to infectious diseases like enteric fever, tuberculosis, roundworm infestation, Meckel's diverticulum, radiation enteritis, Crohn's disease and malignancies like lymphoma, adenocarcinoma and melanoma. ${ }^{(4)}$ Any etiology causing small bowel obstruction and gangrene also leads to perforation.

The importance of early diagnosis and timely management of rupture of intestine cannot be exaggerated-LORD MOYNIHANS(1928). He further emphasized that delay of an hour in any case means that the chances of recovery by operation are thereby reduced ${ }^{(3)}$

Once diagnosed, resuscitation and surgery is the choice of treatment The choice of surgical procedure depends on the general condition of the patient, type of perforation and the state of adjoining bowel segment . Types of surgeries include - Peritoneal drainage , primary closure, resection and anastamosis of the affected bowel segmanet, ileo transverse colostomy, ileostomy and jejunostomy. ${ }^{(5)}$

Even with all improved diagnostic methods and different surgical procedures morbidity and mortality rate still remains high .The post operative complications include wound infection / dehiscence, burst abdomen, eneterocutaneous fistula, intra-peritoneal collection respiratory complications etc .

\section{METHODS:}

This was a prospective observational study conducted in JSS HOSPITAL .In this study, thirty patients who presented with ileo jejunal perforation during the period of one and half years undergoing either primary closure or resection anastamosis were included

\section{Inclusion criteria}

- All patients irrespective of their age or sex presenting to surgical emergency department with acute abdomen, proven to be a case of ileo-jejunal perforation (due to any cause )on basis of operative findings only are to be included in the study.

- Cases where either resection and anastomosis or primary repair are going to be done are to be included in the study

Exclusion criteria

- Cases in which ileostomy is going to be done for ileojejunal perforation
- Cases of hollow viscous perforation other than ileojejunal perforation

- Cases who will not give consent for emergency laparotomy

All data including clinical symptoms, signs, blood and radiological investigations, surgical procedures, postoperative morbidity and mortality, prognosis and its outcome were all collected .The following routine tests including haemoglobin, bleeding time ,clotting time, random blood sugar, serum urea, creatinine, erect $X$ Ray of chest, ECG, peritoneal fluid for culture and sensitivity, pus culture if there was wound infection post operatively were done for all patients .In all perforations other than trauma additional investigations like Widal test and blood Culture were sent.

All of the patients who presented to the casualty were resuscitated with adequate intravenous fluids and intravenous antibiotics. Patients who were not fit to undergo surgery and patients who were initially managed with flank drains under local anaesthesia as a temporary measure to buy time to resuscitate prior to definitive laparotomy were all excluded in the study . Most cases received broad spectrum antibiotics like cephalosporins with metronidazole.

All patients underwent emergency laparotomy under general anaesthesia. The number of perforations ,perforation site and perforation size were all documented .Either of the two surgical procedures were done

1. Simple two layer primary closure

2. Resection and anastomosis

In both the primary closure and resection and anastomosis procedure, the inner layer was closed with 3-0 vicryl and the outer layer was sutured with silk.
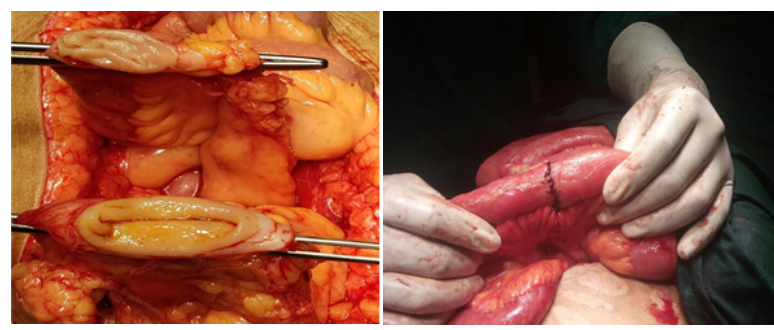

FIGURES 1 and 2 : Showing Resection and anastamosis 
Broad spectrum antibiotics were given post operatively for 5-7 days and was then changed according to culture and sensitivity if needed. If the diagnosis of typhoid was made in that case antibiotics were continued until $10^{\text {th }}$ post operative day.
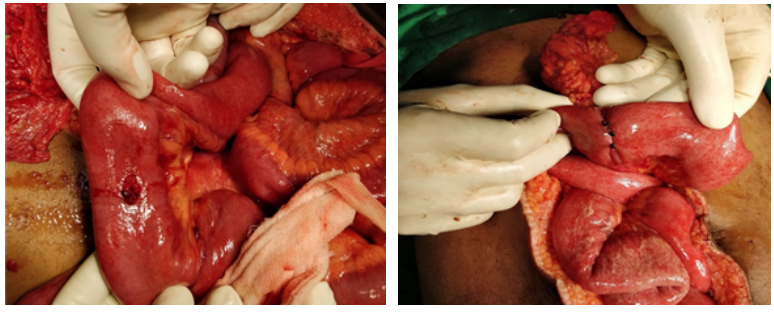

FIGURES 3 and 4 : Showing Primary Closure

Diagnosis of typhoid was made only if the Widal test turned out to be positive (or ) if the organism salmonella was isolated from blood or urine (or) HPE examination shows evidence of typhoid etiology .In case of non-traumatic perforation, investigations were done to identify the aetiology and if no cause was found, it was then termed as nonspecific perforation. Postoperative complications like surgical site infection, burst abdomen, fecal fistula, respiratory complications like effusion, consolidation were all noted.

\section{RESULTS :}

Out of the thirty patients ,commonest cause of jejuno-ileal perforation was Trauma amounting for $40 \%$ of the total. $26.67 \%$ was from blunt trauma and $13.33 \%$ was from penetrating trauma, followed by non specific perforation $26.67 \%$

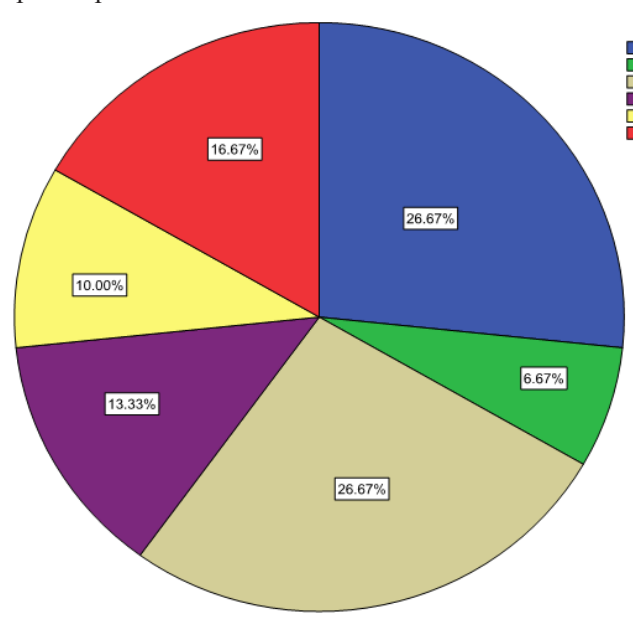

Diagnosis

Blunt Trauma Giverticulitis Nenetrating traum Tuberculosis
Typhoid

FIGURE 5 :Showing incidence of different causes of perforation

The age and sex of the individuals did not influence in deciding the type of surgery and even the incidence of ileo jejunal perforations also did not vary much among different age groups

\begin{tabular}{|c|c|c|c|c|c|}
\hline & \multicolumn{4}{|c|}{ Procedure } \\
\hline & & \multicolumn{2}{|c|}{ Primary repair } & \multicolumn{2}{|c|}{ Resection and Anastamosis } \\
\hline & & $\overline{\text { Coun }}$ & Column N \% & Count & Column N \% \\
\hline \multirow{4}{*}{$\begin{array}{l}\text { Age group in } \\
\text { yrs }\end{array}$} & $<30$ & 5 & $29.4 \%$ & 5 & $38.5 \%$ \\
\hline & $31-50$ & 6 & $35.3 \%$ & 3 & $23.1 \%$ \\
\hline & $51-70$ & 6 & $35.3 \%$ & 5 & $38.5 \%$ \\
\hline & Total & 17 & $100.0 \%$ & 13 & $100.0 \%$ \\
\hline
\end{tabular}

TABLE 1 Showing the Age distribution

\begin{tabular}{|c|l|l|l|l|l|}
\hline \multicolumn{2}{|c|}{} & \multicolumn{3}{|l|}{ Procedure } \\
\cline { 2 - 6 } \multicolumn{2}{|c|}{} & Primary repair & \multicolumn{2}{l|}{ Resection and Anastamosis } \\
\cline { 2 - 6 } \multicolumn{2}{|c|}{} & Count & Column N \% & Count & Column N \% \\
\hline \multirow{3}{*}{ Gender } & Female & 3 & $17.6 \%$ & 3 & $23.1 \%$ \\
\cline { 2 - 6 } & Male & 14 & $82.4 \%$ & 10 & $76.9 \%$ \\
\cline { 2 - 6 } & Total & 17 & $100.0 \%$ & 13 & $100.0 \%$ \\
\hline
\end{tabular}

\section{TABLE 2 Showing the Sex distribution}

The most common presentation of the patients was abdominal pain $(100 \%)$.. Vomitting $(56.7 \%)$ was the second most common symptom .

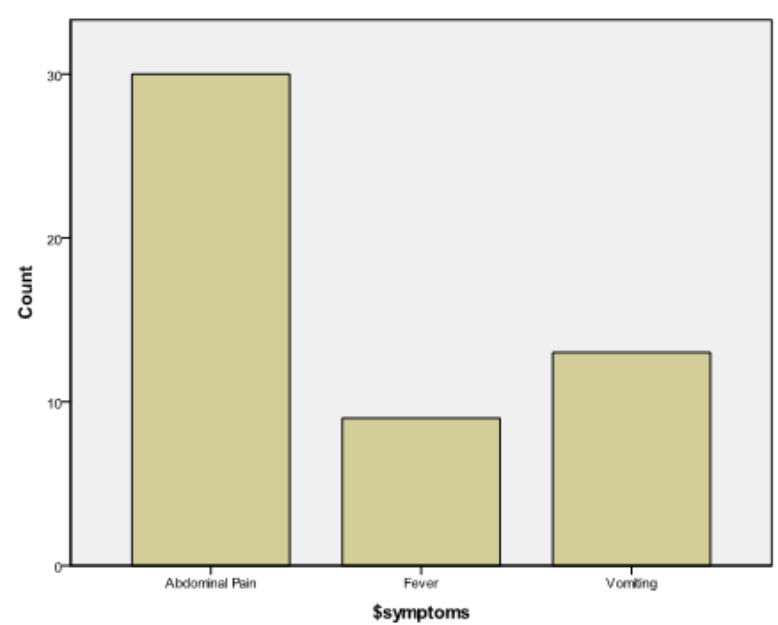

FIGURE 6 :Showing the Symptom presentation

Lag period is the time between time of onset symptoms to time of intervention.Of the nine patients who presented after 5 days of presenting symptom, six patients underwent resection and anastamosis.

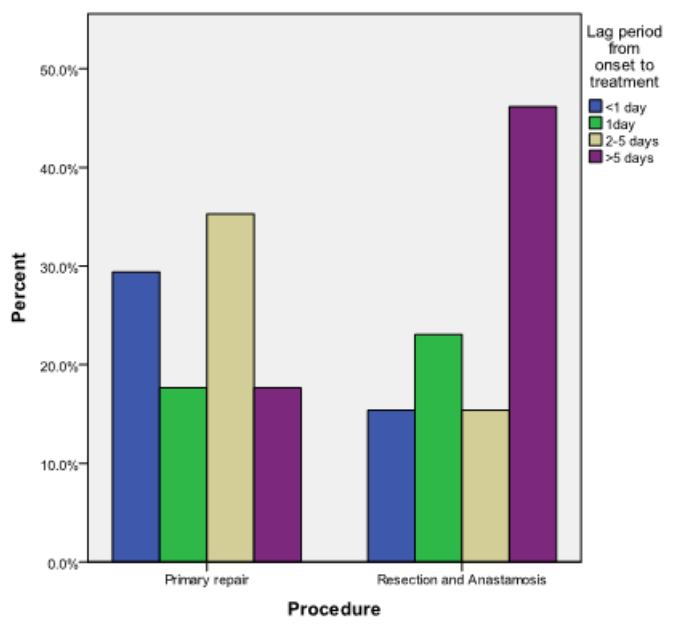

FIGURE 7 : Showing association of Lag period and type of surgery

SIGNS :

Most common sign found at the time of presentation is tenderness of the abdomen .

$86.7 \%$ of the patients had air under diaphragm in the erect $\mathrm{x}$ ray. Shock is another important sign .A total of 11 patients presented with shock and there was not much difference in the number of patients undergoing either of the procedure .

INTRAOPERATIVE FINDINGS :

Depending on the no of perforations,

the size of perforations

the distance from IC junction

The following tables depicts the surgical procedure ,the patients underwent.

\begin{tabular}{|c|c|c|c|c|c|c|c|}
\hline & & \multicolumn{6}{|c|}{ Procedure } \\
\hline & & \multicolumn{2}{|c|}{ Primary repair } & \multicolumn{2}{|c|}{$\begin{array}{l}\text { Resection and } \\
\text { Anastamosis }\end{array}$} & \multicolumn{2}{|l|}{ Total } \\
\hline & & Cou & $\begin{array}{l}\text { Column } \\
\mathrm{N} \%\end{array}$ & Count & $\begin{array}{l}\text { Column } \\
\mathrm{N} \%\end{array}$ & Count & $\begin{array}{l}\text { Column } \\
\mathrm{N} \%\end{array}$ \\
\hline \multirow{4}{*}{$\begin{array}{l}\text { No of } \\
\text { perforations }\end{array}$} & 1.00 & 16 & $94.1 \%$ & 12 & $92.3 \%$ & 28 & $93.3 \%$ \\
\hline & 2.00 & 0 & $.0 \%$ & 1 & $7.7 \%$ & 1 & $3.3 \%$ \\
\hline & 3.00 & 1 & $5.9 \%$ & 0 & $.0 \%$ & 1 & $3.3 \%$ \\
\hline & Total & 17 & $100.0 \%$ & 13 & $100.0 \%$ & 30 & $100.0 \%$ \\
\hline
\end{tabular}

TABLE 4 : No of Perforations and Type of Surgery 
TABLE 5 : Perforation size and Type of Surgery

\begin{tabular}{|c|c|c|c|c|c|c|c|}
\hline & \multicolumn{6}{|c|}{ Size in $\mathrm{cm}$} \\
\hline & & Count & Mean & Standard Deviation & Median & Percentile 25 & Percentile 75 \\
\hline \multirow[t]{3}{*}{ Procedure } & Primary repair & 17 & 1.32 & .81 & 1.00 & 1.00 & 1.00 \\
\hline & Resection and Anastamosis & 13 & 2.08 & .76 & 2.00 & 2.00 & 3.00 \\
\hline & Total & 30 & 1.65 & .86 & 1.00 & 1.00 & 2.00 \\
\hline
\end{tabular}

TABLE 6 : Distance from IC Junction and Type of Surgery

\begin{tabular}{|c|c|c|c|c|c|c|c|}
\hline & \multicolumn{7}{|l|}{ Distance from ICJ in $\mathrm{cm}$} \\
\hline & Count & Mean & Standard Deviation & Median & Percentile 25 & Percentile 75 & \\
\hline \multirow[t]{2}{*}{ Procedure } & Primary repair & 17 & 26.06 & 19.19 & 20.00 & 10.00 & 40.00 \\
\hline & Resection and Anastamosis & 13 & 24.46 & 17.23 & 25.00 & 10.00 & 30.00 \\
\hline
\end{tabular}

\section{COMPLICATIONS}

Post operatively, the most common complication is the wound infection /dehiscence. Out of the 30 patients, eight patients developed it . Out of the eight patients - seven patients had undergone resection and anastamosis. The second most common complication is the respiratory complication and all the patients who had respiratory complication had underwent resection and anastamosis.

TABLE 7 : Incidence of Complications associated with both Surgery

\begin{tabular}{|c|c|c|c|c|c|c|c|c|}
\hline & \multicolumn{6}{|c|}{ Procedure } & \multirow[b]{3}{*}{$\mathrm{p}$} \\
\hline & & \multicolumn{2}{|c|}{ Primary repair } & \multicolumn{2}{|c|}{ Resection and Anastamosis } & \multicolumn{2}{|l|}{ Total } & \\
\hline & & Count & Column N \% & Count & Column N \% & Count & Column N \% & \\
\hline \multirow[t]{2}{*}{ Wound infection/Wound dehiscence } & No & 16 & $94.1 \%$ & 5 & $41.7 \%$ & 21 & $72.4 \%$ & 0.002 \\
\hline & Yes & 1 & $5.9 \%$ & 7 & $58.3 \%$ & 8 & $27.6 \%$ & \\
\hline \multirow[t]{2}{*}{ Burst abdomen } & No & 16 & $94.1 \%$ & 12 & $92.3 \%$ & 28 & $93.3 \%$ & 0.8 \\
\hline & Yes & 1 & $5.9 \%$ & 1 & $7.7 \%$ & 2 & $6.7 \%$ & \\
\hline \multirow[t]{2}{*}{ Entero cutenous fistula } & No & 17 & $100.0 \%$ & 13 & $100.0 \%$ & 30 & $100.0 \%$ & NA \\
\hline & Yes & 0 & $.0 \%$ & 0 & $.0 \%$ & 0 & $.0 \%$ & \\
\hline \multirow[t]{2}{*}{ Respiratory complications } & No & 17 & $100.0 \%$ & 9 & $69.2 \%$ & 26 & $86.7 \%$ & 0.014 \\
\hline & Yes & 0 & $.0 \%$ & 4 & $30.8 \%$ & 4 & $13.3 \%$ & \\
\hline
\end{tabular}

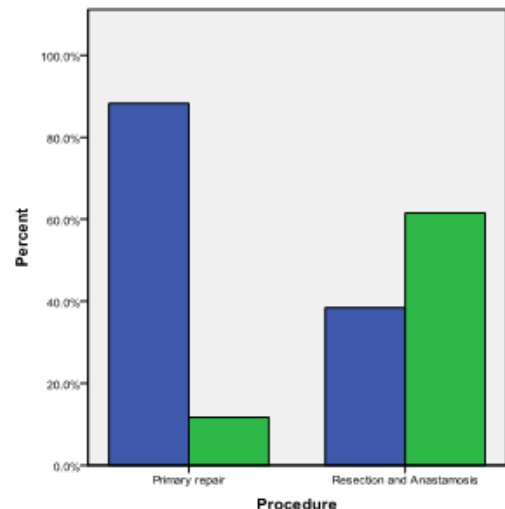

FIGURE 8: Showing Post operative complications associated with both surgery

\section{BASED ON DIAGNOSIS:}

On the basis of etiology, patients with traumatic perforation (both blunt and penetrating ) had underwent primary repair and patients with tuberculosis ,typhoid and diverticulitis had underwent resection and anastamosis.

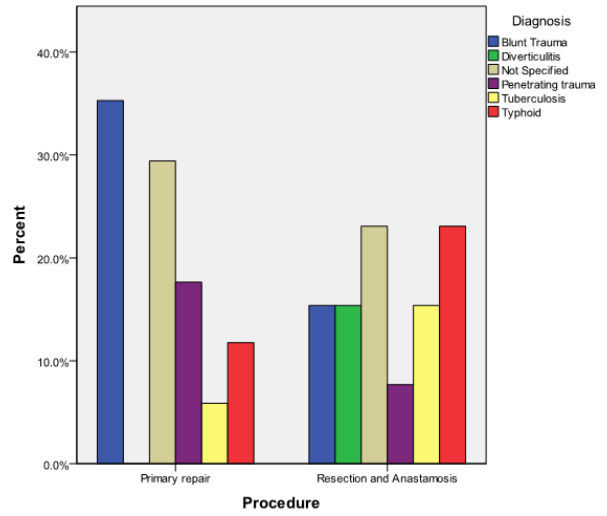

FIGURE 9 : Aetiology and Type of Surgery

\section{DURATION OF HOSPITAL STAY :}

Patients who underwent primary repair had lesser duration of stay when compared to patients who underwent resection and anastamosis.

\section{TABLE 8 : Duration of hospital stay in both surgery}

\begin{tabular}{|l|l|l|l|l|l|l|l|}
\hline \multicolumn{2}{|c|}{} & \multicolumn{5}{|l|}{ Duration of hospital stay in days } \\
\cline { 2 - 7 } \multicolumn{2}{|l}{} & Count & Mean & SD & Median & $\begin{array}{l}\text { Percentile } \\
25\end{array}$ & $\begin{array}{l}\text { Percentile } \\
75\end{array}$ \\
\hline Procedure & $\begin{array}{l}\text { Primary } \\
\text { repair }\end{array}$ & 17 & 15.24 & 3.40 & 15.00 & 13.00 & 16.00 \\
\cline { 2 - 7 } & $\begin{array}{l}\text { Resectio } \\
\text { n and } \\
\text { Anasta } \\
\text { mosis }\end{array}$ & 13 & 17.31 & 3.86 & 16.00 & 14.00 & 18.00 \\
\cline { 2 - 8 } & Total & 30 & 16.13 & 3.69 & 16.00 & 14.00 & 18.00 \\
\hline
\end{tabular}

\section{DISCUSSION:}

Small bowel perforations is one of the commonest surgical emergency in our developing nation. The commonest cause of ileo -jejunal perforation in this study was trauma. A total of $40 \%$ patients presented with history of trauma of which $26.7 \%$ comprises of blunt trauma and remaining was penetrating trauma, followed by non specific causes $(26.7 \%)$ and then typhoid (13.3\%).

According to the study by Nahar $\mathrm{S}$ et al, in jejunal perforation, blunt trauma was the commonest cause and in ileal perforation enteric fever was the commonest cause ${ }^{(3)}$

The mean age in the study by Nahar S et al was 34.37 years ${ }^{(3)}$ which was close to mean age of 33.72 years reported by Shah $\mathrm{S}$ and Gandhi JP et $\mathrm{al}^{\left({ }^{(6)}\right.}$. But in contrast to all that in this study, maximum no of patients were found to be in age group of 51-70 yrs.

In this study, ileo jejunal perforations are more in males with a ratio of $4: 1$. The male to female ratio in studies like Poornima $\mathrm{R}$ et al and Shrivastava $\mathrm{D}$ et al were $4.33: 1$ and $6.38: 1$ respectively which was similar to our results. ${ }^{(7)}$

Clinical course of the disease usually starts with bacteremia with highgrade fever proceeding to systemic sepsis which is characterised by normal or low leukocyte counts and anemia. Pain abdomen was the most common symptom in cases of perforation and was seen in $100 \%$ of our cases. This was also shown in studies by Poornima R et al Singla et al and Khalid S et al. In Khalid S et al study of 125 cases ,pain abdomen was present in $100 \%$ of patients. ${ }^{(8)}$

Among the signs elicited in the patients presenting with perforation , most common is the diffuse tenderness with $100 \%$ incidence, rigidity and guarding were found in $80 \%$ of cases and patients presenting with shock were $36 \%$ in this study. Singla et al study also reveals that as far physical signs were concerned, abdominal tenderness was present in all patients of the study. 
Erect X-ray chest or abdomen displaying both domes of diaphragm is a cheap and useful investigation to detect hollow viscus perforation. In this study $86.7 \%$ presented with air under diaphragm. This is supported by other studies like JP Singh et al (50-70\%) ${ }^{(6)}$ and Khalilur RAet al $(71.4 \%)^{(9)}$

Of the thirty patients in this study, seventeen underwent primary repair and rest thirteen underwent resection and anastamosis. Based on the etiology, trauma patients mostly have undergone primary repair and in patients with underlying infection like typhoid, TB and tuberculosis, resection and anastamosis was more commonly done

Depending on the lag period, when the time interval is more than 5 days, the incidence of resection and anastamosis was high $46.2 \%$ when compared to primary repair $17.6 \%$

The most common complication following surgery for ileo jejunal perforations in this study was wound infection/dehiscence with an incidence of $27.6 \%$. Studies by Nahar S et al ${ }^{(3)}$ and Khalilur RA et al ${ }^{(9)}$ also provide evidence regarding wound infection being the most common complication.In this study among the eight patients who suffered from wound infection/dehiscence, seven patients had undergone resection and anastamosis. The second most common complication is the respiratory complications and all the four patients in this study underwent resection and anastamosis.

By logistic regression the risk of any complications is twenty nine times higher in resection and anastamosis group compared to primary repair after adjusting for perforation size and distance from ICJ .

TABLE 9. : SHOWING COMPARISON OF VARIOUS STUDIES ON RATE OF COMPLICATIONS :

\begin{tabular}{|l|l|l|l|l|}
\hline & $\begin{array}{l}\text { Wound } \\
\text { infection/dehiscence }\end{array}$ & $\begin{array}{l}\text { Respiratory } \\
\text { complications }\end{array}$ & $\begin{array}{l}\text { Burst } \\
\text { abdomen }\end{array}$ & ECF \\
\hline My study & $27.6 \%$ & $13.3 \%$ & $6.7 \%$ & 0 \\
\hline $\begin{array}{l}\text { Nahar S et } \\
\text { al }\end{array}$ & $68.41 \%$ & $15.78 \%$ & 0 & $10.52 \%$ \\
\hline $\begin{array}{l}\text { Sharath S } \\
\text { et al }\end{array}$ & $34 \%$ & - & $6 \%$ & $2 \%$ \\
\hline $\begin{array}{l}\text { Sudhersha } \\
\text { nkapoor et } \\
\text { al }\end{array}$ & $70 \%$ & $35 \%$ & $12 \%$ & $8 \%$ \\
\hline
\end{tabular}

\section{CONCLUSIONS :}

According to this study, risk of complications following resection and anastamosis was twenty nine times higher in resection and anastamosis group compared to primary closure after adjusting for perforation size and distance from IC junction Furthermore, if the lag period, (ie) the time between onset of symptoms and time of presentation, was less, the chance of patient needed to undergo resection and anastamosis was also less which further implied that the post operative morbidity was the less in those patients.. Mean duration of hospital stay was also longer in resection and anastamosis group (17 days ) compared to primary repair group.

Based on this study, we would like to conclude that the morbidity following resection and anastamosis was more when compared to primary closure after considering most of the pre operative and intra operative factors, hence primary closure should always be preferred in a clinically stable patient with healthy bowel .

\section{REFERENCES :}

1. Mittal, S., Singh, H., Munghate, A., Singh, G., Garg, A., \& Sharma, J. (2014). A comparative study between the outcome of primary repair versus loop ileostomy in ileal perforation. Surgery research and practice, 2014.

2. Pal, D. K. (1998). Evaluation of best surgical procedures in typhoid perforation - an experience of 60 cases. Tropical doctor, 28(1), 16-18.

3. Nahar, S., \& Ranjan, A. (2017). Observational study of small bowel perforation in a tertiary care hospital. International Surgery Journal, 4(8), 2746-2750.

4. Kapoor, S., Varma, V., Mehta, N., Kumaran, V., \& Nundy, S. (2017). Study of surgical complications of exploratory laparotomy and their management-a study of 100 cases. IOSR J Dent Med Sci, 16, 36-41.

5. Madhu K., Study of Different Surgical Management And Their Complication Rates in Ileal Perforation. (2017). IOSR Journal of Dental and Medical Sciences (IOSR-JDMS), 16(8), 51-56.

6. Gandhi, J. P., \& Shah, S. (2015). Role of ileostomy in enteric perforation. IJSS Journal of Surgery, 1(1), 10-15.

7. Poornima, R., Venkatesh, K. L., Goutham, M. V., \& Hassan, N. (2017) Clinicopathological study of Ileal perforation: study in tertiary center. International Surgery Journal, 4(2), 543-549.

8. Khalid, S., \& Bhatti, A. A. (2014). Non-traumatic spontaneous ileal perforation: experience with 125 cases. Journal of Ayub Medical College Abbottabad, 26(4), 526-9.

Rahman, K., Krishnaswamy, J., Muthukumaran, G., \& Prakash, S. (2018). A comparative study on outcome of ileal perforation after primary perforation closure and 